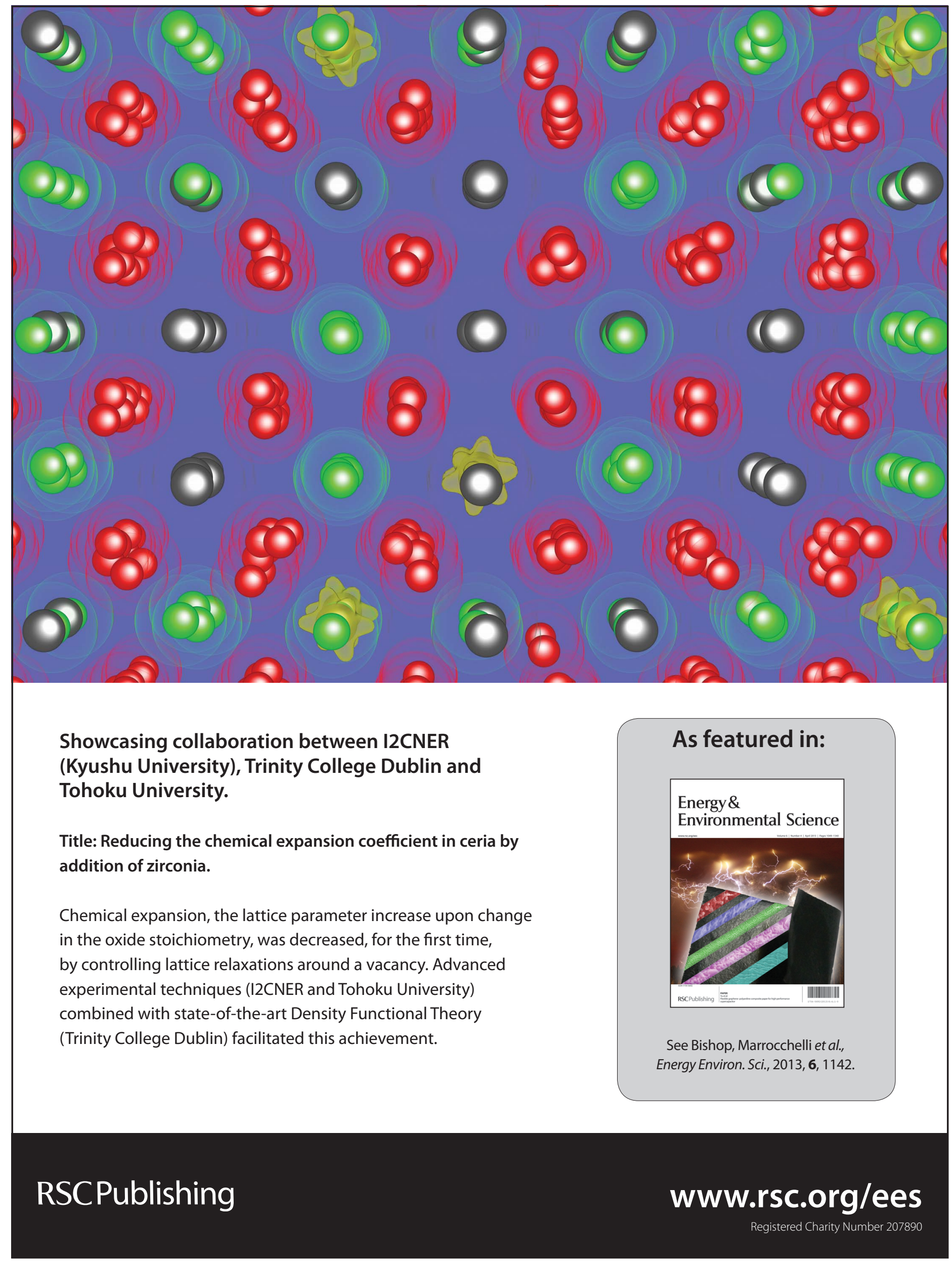




\section{Reducing the chemical expansion coefficient in ceria by addition of zirconiat}

Cite this: Energy Environ. Sci., 2013, 6, 1142

Received 28th October 2012

Accepted 1st February 2013

DOI: $10.1039 / c 3 e e 23917 a$

\author{
S. R. Bishop, $\neq^{\star a b}$ D. Marrocchelli, $\ddagger^{\star c}$ W. Fang, ${ }^{d}$ K. Amezawa, ${ }^{d}$ K. Yashiro ${ }^{d}$ \\ and G. W. Watson ${ }^{\mathrm{C}}$
}

www.rsc.org/ees

For the first time, the role of isovalent $Z r$ substitution in cerium oxide on the non-stoichiometry induced dilation (chemical expansion) was investigated. Chemical expansion was derived experimentally using HTXRD, dilatometry, and TGA measurements on $\operatorname{Pr}_{0.1} \mathrm{Zr}_{0.4} \mathrm{Ce}_{0.5} \mathrm{O}_{1.95-\delta}$ and computationally with DFT calculations on $\mathrm{Zr}_{0.5} \mathrm{Ce}_{0.5} \mathrm{O}_{2-\delta}$. Though $\mathrm{Zr}$ was found to increase the reducibility and the corresponding chemical expansion of ceria in the studied range, the relationship between chemical expansion and non-stoichiometry (the chemical expansion coefficient) was significantly smaller ( $54 \%$ less) than that observed in ceria, and consistent with predictions from the authors' previous work. The origin of the reduced chemical expansion coefficient, associated with a larger contraction of the lattice around oxygen vacancies, is explained using DFT calculations and corroborated with prior investigations of enhanced reducibility of ceriazirconia. Additionally, implications of this discovery for solid oxide fuel cells and heterogeneous catalyst systems are also discussed.

Oxygen non-stoichiometric oxides are commonly used in solid oxide fuel cells (SOFCs), where the simultaneous presence of large concentrations of oxygen vacancies and charge compensating electrons lead to mixed ionic electronic conductivity. This serves to both increase the active area of SOFC electrodes for oxygen reduction and fuel oxidation, as well as reduce the barriers for such reactions. ${ }^{1}$ Due to the intrinsic nature of the defects, changes in temperature and oxygen partial pressure (typical of the environment in a SOFC) often lead to large changes in oxygen stoichiometry and, correspondingly, a large defect-

${ }^{a}$ International Institute for Carbon Neutral Energy Research (WPI-I2CNER), Kyushu University, Nishi-ku Fukuoka 819-0395, Japan. E-mail: bishop@i2cner.kyushu-u.ac.jp ${ }^{b}$ Department of Materials Science and Engineering, Massachusetts Institute of Technology, Cambridge, MA 02139, USA

${ }^{c}$ School of Chemistry, Trinity College Dublin, Dublin 2, Ireland. E-mail: marroccd@ tcd.ie

${ }^{d}$ Institute of Multidisciplinary Research for Advanced Materials, Tohoku University, Sendai 980-8577, Japan

$†$ Electronic supplementary information (ESI) available. See DOI: 10.1039/c3ee23917a

‡ These authors contributed equally to this manuscript.

\section{Broader context}

Oxygen non-stoichiometric oxides, e.g. $\mathrm{CeO}_{2}$ and $\mathrm{TiO}_{2}$, are routinely used in energy applications, such as solid oxide fuel cells, three-way catalysts, Li-ion batteries and water splitting. Chemical expansion is the lattice parameter increase caused by changes in the oxygen content of these materials and it has been recognized as a significant source of device degradation and subsequent failure. This paper represents the first successful attempt to minimize chemical expansion through a novel technique of controlling lattice relaxation around atomic level defects in $\mathrm{CeO}_{2}$. This was possible thanks to a detailed understanding of the atomistic factors responsible for chemical expansion, obtained previously by the authors using a combined computer simulation and experimental approach. The development reported in our paper will aid researchers to create systems where chemical expansion can be mitigated or even tuned, according to specific device needs.

induced dilation, known as chemical expansion..$^{2,3}$ As an example, the high ionic conductivity SOFC electrolyte and anode material, $\mathrm{Gd}_{x} \mathrm{Ce}_{1-x} \mathrm{O}_{2-x / 2-\delta}$, is known to become highly oxygen deficient $\left(\sim 4 \%\right.$ at $\left.800{ }^{\circ} \mathrm{C}\right)$ under anode conditions with isothermal chemical expansion on the order of $1-2 \% .^{4-6}$ In order to avoid mechanical failure, such behavior places constraints on the operational range and design of SOFCs. ${ }^{3,7-9}$ Additionally, oxygen storage materials, used in automotive three-way-catalysts, undergo rapidly changing operating conditions with consequent cyclic chemical expansion, resulting, in some cases, in their fracture. ${ }^{10}$ These materials present large structural relaxations, localized primarily around the smaller cation species, which dramatically decrease the vacancy formation energy and thus enhance oxide reducibility, a key figure of merit. ${ }^{11}$ Other examples of this phenomenon are the sometimes dramatic electrode expansion in Li-ion batteries during $\mathrm{Li}$ intercalation ${ }^{12,13}$ and the strain-induced metallic to insulator transitions in superconductors, ${ }^{\mathbf{1 4}}$ with rearrangement of ions around point defects.

Recently, using a combination of advanced simulation and empirical analysis techniques, the authors showed that, in $\mathrm{CeO}_{2}$ and $\mathrm{ZrO}_{2}$, the chemical expansion coefficient $\left(\alpha_{\mathrm{C}}\right)$, analogous to the thermal expansion coefficient, is the result of two 
competing processes. The first one is the formation of an oxygen vacancy, with surrounding lattice contraction (primarily due to electrostatic interactions), and the second is a change in the cation radius, resulting in lattice expansion (primarily due to steric effects), ${ }^{15,16}$ with the latter contribution much larger than the former and thus resulting in a net expansion upon reduction of the material. The importance of charge localization was also recognized in a subsequent study ${ }^{17}$ An interesting result of our investigation was that the contraction due to the formation of an oxygen vacancy in $\mathrm{ZrO}_{2}$ (upon acceptor doping) is $91 \%$ larger than in $\mathrm{CeO}_{2}$, leading to our prediction that a $\mathrm{CeO}_{2}-\mathrm{ZrO}_{2}$ solid solution will have a smaller chemical expansion coefficient. ${ }^{16}$ In this communication, we test this hypothesis, both experimentally and computationally, and show that the solid solution indeed exhibits a significant $(\sim 50 \%)$ decrease in $\alpha_{\mathrm{C}}$. Implications of this discovery will also be discussed.

Expansion measurements, performed using dilatometry or high temperature X-ray diffraction (HTXRD) on dense or powder samples, respectively (experimental details are reported in the ESI $\dagger$ ), are shown in Fig. 1a for $\mathrm{Pr}_{0.1} \mathrm{Zr}_{0.4} \mathrm{Ce}_{0.5} \mathrm{O}_{1.95-\delta}$ (PZCO) and compared with data in the literature for $\mathrm{Gd}_{0.1} \mathrm{Ce}_{0.9} \mathrm{O}_{1.95-\delta}$ (GCO). With decreasing $\mathrm{pO}_{2}$ at each temperature, both GCO and PZCO exhibit expansion (with the isothermal expansion defined as zero in air at each temperature). At each temperature, the expansion of PZCO with decreasing $\mathrm{pO}_{2}$ is greater than GCO, seemingly contradicting the earlier predictions of reduced $\alpha_{\mathrm{C}}$ and an observation we will come back to later.

Turning to the non-stoichiometry data (measured by thermogravimetry), one observes that during reduction, both PZCO and GCO become more non-stoichiometric (with $\delta \cong 0$ in air), consistent with the following reaction (written in Kröger-Vink notation $^{18}$ ).

$$
\mathrm{O}_{\mathrm{O}}^{\times} \leftrightarrow V_{\mathrm{O}}^{*}+2 \mathrm{e}^{\prime}+\frac{1}{2} \mathrm{O}_{2}(\mathrm{~g})
$$

where $\mathrm{O}_{\mathrm{O}}^{\times}$is an oxide ion on its respective lattice site, $V_{\mathrm{O}}^{*}$ is an oxygen vacancy with a double positive charge with respect to the lattice, and $\mathrm{e}^{\prime}$ is an electron (localized on a cerium cation ${ }^{19}$ ). It is clear from the figure that $\delta$ (on the log scale) is significantly larger in PZCO than in GCO, consistent with the well-known decrease in the vacancy formation energy upon $\mathrm{Zr}$ substitution, discussed later. In fact, the enhanced reducibility has lead to full reduction of Pr to the trivalent state, even in air conditions (see discussion of Fig. A in ESI $\dagger$ and $c f .{ }^{21}$ ) making it a trivalent acceptor dopant $\left(\right.$ like $\mathrm{Gd}^{3+}$ ) throughout the studied temperature and $\mathrm{pO}_{2}$ range. In order to decouple the differences of $\delta$ in these two materials, the chemical expansion coefficient is now extracted as discussed below.

The isothermal chemical expansion coefficient $\left(\alpha_{\mathrm{C}}\right)$ linearly defines the relationship between chemical expansion $\left(\varepsilon_{\mathrm{C}}\right)$ and change in oxygen non-stoichiometry $(\Delta \delta)$ as described by the equation below: ${ }^{2}$

$$
\varepsilon_{\mathrm{C}}=\alpha_{\mathrm{C}} \Delta \delta
$$

Using the expansion and non-stoichiometry data in Fig. $1, \alpha_{\mathrm{C}}$ is derived from the slope of $\varepsilon_{\mathrm{C}}$ versus $\delta$ in Fig. 2. The slope is
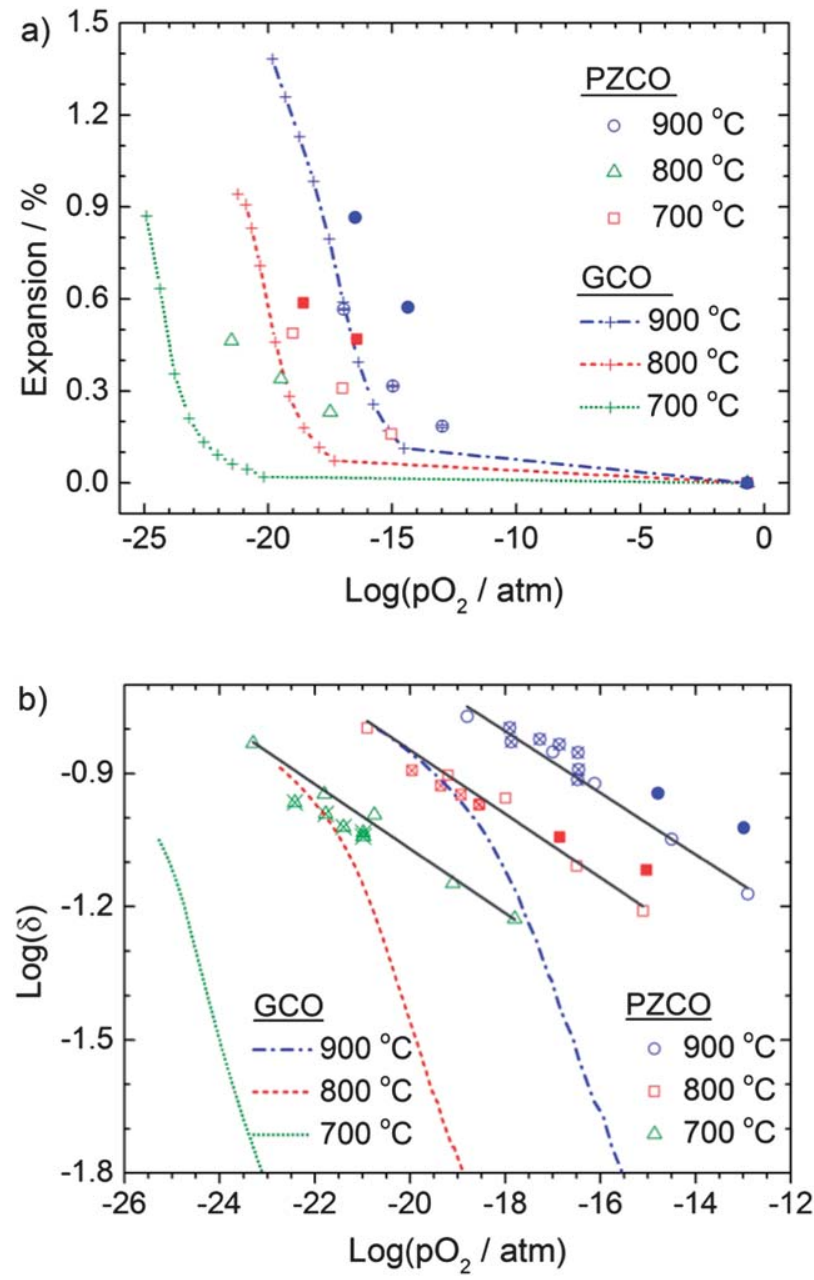

Fig. 1 Isothermal chemical expansion (a) and oxygen non-stoichiometry (b) plotted against oxygen partial pressure. Closed symbols are bulk TGA and dilatometry data and open symbols are powder TGA and HTXRD data (symbols with $\times$ 's indicate a second set of powder data) for $\operatorname{Pr}_{0.1} \mathrm{Zr}_{0.4} \mathrm{Ce}_{0.5} \mathrm{O}_{1.95-\delta}$ (PZCO). Dotted and dashed lines are from the literature for $\mathrm{Gd}_{0.1} \mathrm{Ce}_{0.9} \mathrm{O}_{1.95-\delta}(\mathrm{GCO}) .{ }^{4}$ Solid lines represent linear fits to the first set of powder PZCO data, used with expansion data to extract the stoichiometry expansion relationship shown in Fig. 2.

significantly less in PZCO than in GCO, clearly demonstrating that the increase in volume with increase in non-stoichiometry $(\delta)$ is smaller in PZCO. This also shows that the greater chemical expansion of $\mathrm{PZCO}$ for a given change in $\mathrm{pO}_{2}$ (Fig. 1a) only arises because PZCO is more easily reduced than GCO. The average $\alpha_{\mathrm{C}}$ for all the PZCO experimental data is $0.046 \pm 0.01$, in good agreement with simulated DFT data $\left(\alpha_{\mathrm{C}}=0.048\right.$, see below) and is $54 \%$ less than in $\operatorname{GCO}\left(\alpha_{\mathrm{C}}=0.101\right) .{ }^{22}$ Additionally, $\alpha_{\mathrm{C}}$ in PZCO is $45 \%$ less than in $\operatorname{Pr}_{0.1} \mathrm{Ce}_{0.9} \mathrm{O}_{2-\delta}\left(\alpha_{\mathrm{C}}=0.087 \pm 0.005\right)$ indicating that the difference in $\alpha_{\mathrm{C}}$ is not due to choice of trivalent Gd or Pr doping. ${ }^{23}$ Values of $\alpha_{\mathrm{C}}$ for each set of data are reported in Table 1 of the ESI. $†$

Following the same procedure used in our previous work, the effective radius of an oxygen vacancy is calculated using the following equation. ${ }^{16}$

$$
r_{V}=r_{\mathrm{O}}+4\left(r_{\mathrm{h}}+r_{\mathrm{O}}\right)\left(\alpha_{\mathrm{C}}-\alpha_{\mathrm{M}}\right)
$$




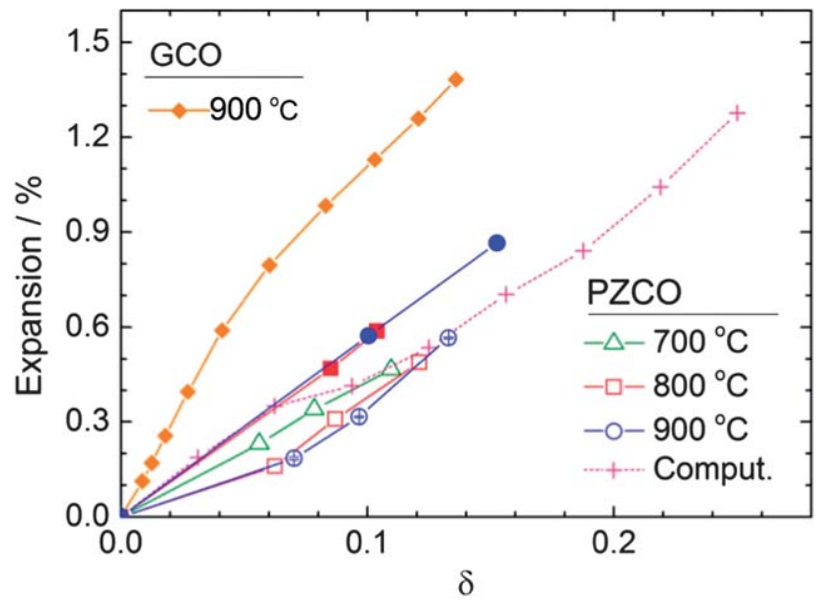

Fig. 2 Expansion versus non-stoichiometry, where the slope of the data is the chemical expansion coefficient. Data for GCO are from ref. 4. For PZCO, closed points are from dense samples (dilatometry) and open points are from powder (HTXRD). Plus symbols are from computational predictions for $\mathrm{Zr}_{0.5} \mathrm{Ce}_{0.5} \mathrm{O}_{2-\delta}$.

where $r_{\mathrm{h}}$ is the host cation radius (taken, in this case, as the weighted average radius between $\mathrm{Ce}^{4+}, \mathrm{Pr}^{3+}$, and $\mathrm{Zr}^{4+}=$ $0.9336 \AA) ; r_{\mathrm{O}}$ the oxygen radius $(1.38 \AA)$; and $\alpha_{\mathrm{M}}$ the expansion due to cation radius change. The average value of $r_{V}=0.966 \pm$ $0.092 \AA$ calculated from the experimental data for PZCO (see Table 2, ESI $\dagger$ ) is in excellent agreement, within error, to the one obtained in our previous work for $\mathrm{ZrO}_{2}, r_{V}=0.988 \pm 0.039 \AA$ and much smaller than that for $\mathrm{CeO}_{2}\left(r_{V}=1.169 \pm 0.059 \AA\right)$, indicating that $\mathrm{Zr}$ plays a key role in decreasing $\alpha_{\mathrm{C}}$, discussed in the next section.

As shown in Fig. 2, the DFT calculations on fluorite-structured ${ }^{24} \mathrm{Zr}_{0.5} \mathrm{Ce}_{0.5} \mathrm{O}_{2-\delta}$ (ZCO) with different oxygen stoichiometries are in very good agreement with the experimental results (computational details are reported in the ESI $\dagger$ ). Here we note that, since the chemical expansion coefficient has been shown to be approximately temperature independent, ${ }^{4,23}$ we do not foresee a discrepancy between the $0 \mathrm{~K}$ DFT calculations and the elevated temperature measurements. To simplify the modeling, Pr was not included in the studied composition, and is not expected to significantly alter the calculation, as it is has a fixed valence in the system (as discussed above). In addition to predicting macroscale properties, verifiable by experiment, an advantage of the computational technique is its insight into the local atomic structure, such as the relaxation pattern of ions around defects. For instance, the importance of local relaxations has already been recognized by Wang et al. in the context of the outstanding oxygen storage capacity of $\kappa-\mathrm{Ce}_{2} \mathrm{Zr}_{2} \mathrm{O}_{8}{ }^{11}$

In Fig. 3, atomic positions of the ions close to a vacancy are shown with grey arrows indicating direction of displacements (with respect to the perfect crystal) greater than $0.20 \AA$ and small black arrows indicating bond distances. It is immediately apparent that not all displacements are equal, nor in the same direction, i.e. that the introduction of undersized $\mathrm{Zr}$ distorts and destabilizes the oxygen sub-lattice, as indicated by Lee et $a .^{25}$ In general, cations are repelled by the vacancy and anions attracted to it, consistent with our prior investigation of pure $\mathrm{CeO}_{2}$, with the magnitude of contraction due to anion

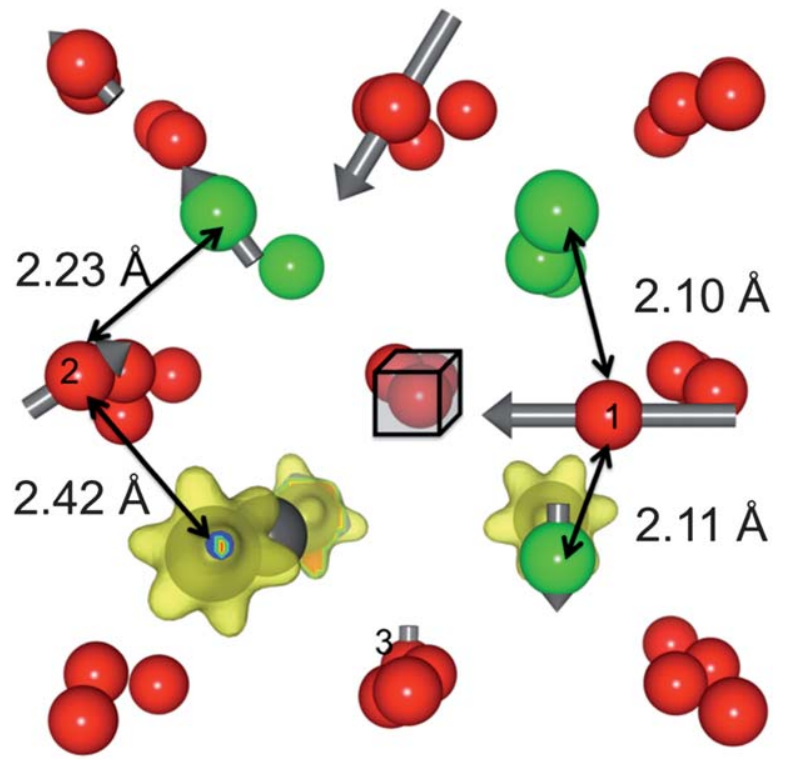

Fig. 3 Perspective image of the local relaxations around a vacancy in ZCO. Red, green and grey spheres are oxygen, zirconium and cerium atoms, respectively. Also shown are the $4 \mathrm{f}$ electron charge density (yellow), indicating where $\mathrm{Ce}^{3+}$ cations reside in the lattice. Due to periodic boundary conditions, the same cation can appear twice in the lattice, as is the case for the $\mathrm{Ce}^{3+}$ cations in the bottom-left row. The grey, thick arrows indicate atomic displacements greater than $0.2 \AA$, with the arrow length corresponding to twice the displacement of the ion. Selected bond distances (black, thin arrows) are also reported.

displacements greater than expansion from cation displacements, leading to overall lattice contraction around a vacancy. ${ }^{16}$ In the Figure, the presence of $\mathrm{Zr}$ causes a large variation in displacements among the anions, with a dependence on their nearest neighbor cations. The oxide ion labeled 1 (see Fig. 3) is strongly displaced towards the vacancy (to the left and into the plane of the paper) by $0.53 \AA$, more than twice the displacement $(0.25 \AA$ ) of oxide ion 2 (and much greater than the average displacement of $\sim 0.16 \AA$ found for pure ceria ${ }^{16}$ ). This behavior arises because oxide ion 1 is bound to two small $\mathrm{Zr}^{4+}$ ions, allowing greater displacement as compared to oxide ion 2 , which is bound to a $\mathrm{Zr}^{4+}$ ion and a larger $\mathrm{Ce}^{3+}$ ion that creates a stronger steric hindrance, blocking the oxide ion from further displacement toward the vacancy. This displacement hindrance is confirmed by the longer bond distances between the oxide, $\mathrm{Ce}^{4+}$ and $\mathrm{Ce}^{3+}$ ions (2.20-2.50 $\AA$ on average) as compared to oxide and $\mathrm{Zr}^{4+}$ ions (2.04-2.18 $\AA$ on average). In the extreme case of two $\mathrm{Ce}^{3+}$ ions blocking an oxide ion (labelled 3 in Fig. 3), the oxide ion is, counter intuitively, displaced $0.21 \AA$ away from the vacancy, indicating the importance of complex interactions among all the ions as a whole in this solid solution. Overall, the presence of $\mathrm{Zr}$ near a vacancy leads to shorter oxide ion to $\mathrm{Zr}^{4+}$ ion bond lengths (in agreement with previous calculations ${ }^{11}$ ) with a correspondingly larger contraction, and hence smaller chemical expansion than pure ceria. By controlling the lattice relaxation around an oxygen vacancy, this novel solid solution strategy presents a new way to reduce chemical expansion.

The markedly enhanced reducibility for ZCO, as observed here and in several prior investigations, ${ }^{20,26-28}$ is believed to 
originate from local distortions of the lattice around $\mathrm{Zr}$ in an attempt to accommodate the relatively small $\mathrm{Zr}$ size. ${ }^{25,29-31}$ With formation of vacancies, Zr obtains a lower coordination number, aiding in stabilizing the ZCO solid solution in a similar fashion as acceptor-doped, cubic-fluorite-stabilized zirconia. ${ }^{25,32-34}$ Indeed, this localized distortion tendency is confirmed in the present expansion study, since $\alpha_{\mathrm{C}}$ (and $r_{V}$ ) for PZCO is found to be very similar to that for cubic $\mathrm{ZrO}_{2}$, suggesting that vacancies are associated with $\mathrm{Zr}$ cations as opposed to adopting a random distribution with an expected $\alpha_{\mathrm{C}}$ (and $r_{V}$ ) between that for $\mathrm{CeO}_{2}$ and $\mathrm{ZrO}_{2}$. This results in significantly larger lattice distortions and oxygen displacements towards the vacancy near $\mathrm{Zr}$ (shown in Fig. 3) as compared to our previous study on undoped ceria ${ }^{16}$ and in agreement with a computational study from Wang et al. predicting that such localized oxygen displacements lead to the outstanding oxygen storage capacity and release in $\kappa-\mathrm{Ce}_{2} \mathrm{Zr}_{2} \mathrm{O}_{8}$, a similar material. ${ }^{11}$

As an illustration of the impact of this result, we present the following example. The reduced chemical expansion coefficient of PZCO and ZCO solid solutions has important ramifications in SOFC electrodes as well as three-way catalysts for automotive applications, where the oxygen storage capacity of ZCO serves as a buffer for redox reactions. ${ }^{\mathbf{1 0 2 0}}$ In both cases, mechanical stability on redox cycling is important, as well as the enhanced oxygen deficiency, or storage capacity. For example, with changes in power demand or during initial startup, large changes in oxygen activity at an SOFC anode occur as more or less steam is produced or the cell is exposed to fuel gas for the first time, respectively, resulting in changes in $\delta$ and hence expansion. Upon first exposing the cell to typical anode testing conditions $\left(\mathrm{H}_{2}\right.$ with $3 \%$ water at $\left.800{ }^{\circ} \mathrm{C}, \mathrm{pO}_{2} \sim 10^{-21.3} \mathrm{~atm}\right)$ from $\mathrm{O}_{2}$, both GCO and PZCO will expand, with PZCO initially expanding more (see Fig. 1a), until the final oxygen activity is reached whence expansion of GCO and PZCO are comparable $(\sim 0.8 \%$, calculated using extrapolation of PZCO $\delta$ data to the low $\mathrm{pO}_{2}$ ). Now, for a change in steam content from $3 \%$ to $50 \%$, under the otherwise same conditions (final $\mathrm{pO}_{2} \sim 10^{-18.3} \mathrm{~atm}$ ), the corresponding chemical expansion for PZCO is $-0.3 \%$, less than half that for GCO $(-0.7 \%)$ and presumably leading to improved cyclability during operation. Of course, the impact of $\mathrm{Zr}$ on other properties, such as the reported decrease in ionic conductivity in $\mathrm{ZCO},{ }^{35,36}$ are important factors to consider when designing devices. Notwithstanding this, recent work on freestanding thin-film SOFCs successfully employed a zirconiaceria electrolyte, with improved mechanical properties over pure ceria and with relatively high power density. ${ }^{37}$

\section{Conclusions}

In conclusion, we reported HTXRD and dilatometry measurements of the chemical expansion of $\operatorname{Pr}_{0.1} \mathrm{Zr}_{0.4} \mathrm{Ce}_{0.5} \mathrm{O}_{1.95-\delta}$. We find that, despite its easier reducibility, this material presents a significantly smaller chemical expansion coefficient compared to that observed in ceria. This is consistent with predictions from our previous work on chemical expansion in $\mathrm{CeO}_{2}$ and $\mathrm{ZrO}_{2}{ }^{16}{ }^{16}$ DFT calculations were performed on $\mathrm{Zr}_{0.5} \mathrm{Ce}_{0.5} \mathrm{O}_{2-\delta}$ to understand the atomistic sources of this phenomenon. It was found that oxide ions close to a vacancy are strongly displaced towards it, and these displacements are facilitated by the smaller ionic radius of the $\mathrm{Zr}^{4+}$ cations. Their presence, therefore, results in a bigger contraction of the lattice around the vacancy, that, in turn, better compensates the cation expansion upon reduction, resulting in a smaller chemical expansion coefficient than ceria without zirconia. This behavior coincides with prior investigations indicating that localized lattice relaxations around $\mathrm{Zr}$ cations, facilitated by oxygen vacancies, leads to increased reducibility. This demonstration of controlling the lattice relaxation around a vacancy offers a new approach towards decreasing the chemical expansion coefficient of non-stoichiometric oxides.

\section{Acknowledgements}

SRB acknowledges support from the Advanced Low Carbon Technology Research and Development Program (ALCA) funded by JST, Japan; WPI-I2CNER supported by the World Premier International Research Initiative (WPI), MEXT, Japan; and the Basic Energy Sciences, U. S. Department of Energy (award DESC0002633). DM wishes to thank the Government of Ireland for an EMPOWER Postdoctoral Fellowship and the Trinity Centre for High Performance Computing for computing time. GWW would like to thank SFI for funding (grant numbers 08/RFP/ MTR1044 and 09/RFP/MTR2274). The SFI/HEA Irish Centre for High-End Computing (allocations tcche026b and tcche031b) is also thanked for the provision of computational facilities and support. The authors would like to thank Profs. Bilge Yildiz (MIT) and Harry L Tuller (MIT) for stimulating discussions and previous work on chemical expansion in ceria.

\section{Notes and references}

1 S. B. Adler, Chem. Rev., 2004, 104, 4791.

2 S. B. Adler, J. Am. Ceram. Soc., 2001, 84, 2117.

3 A. Atkinson and T. Ramos, Solid State Ionics, 2000, 129, 259.

4 S. R. Bishop, K. L. Duncan and E. D. Wachsman, Acta Mater., 2009, 57, 3596.

5 S. Hull, et al., J. Solid State Chem., 2009, 182, 2815.

6 M. Mogensen, et al., Solid State Ionics, 2004, 174, 279.

7 Y. Wang, et al., Solid State Ionics, 2007, 178, 53.

8 B. Sheldon and V. Shenoy, Phys. Rev. Lett., 2011, 106, 216104.

9 K. Sato, et al., J. Test. Eval., 2006, 34, 246.

10 M. Sugiura, Catal. Surv. Asia, 2003, 7, 77.

11 H.-F. Wang, et al., Angew. Chem., Int. Ed., 2009, 48, 8289.

12 Y. M. Chiang, Science, 2010, 330, 1485.

13 W. H. Woodford, Y. M. Chiang and W. C. Carter, J. Electrochem. Soc., 2010, 157, A1052.

14 Y. Kuru, et al., J. Cryst. Growth, 2010, 312, 2904.

15 M. Burbano, et al., J. Phys.: Condens. Matter, 2011, 23, 255402.

16 D. Marrocchelli, et al., Adv. Funct. Mater., 2012, 22, 1958.

17 D. Marrocchelli, et al., Phys. Chem. Chem. Phys., 2012, 14, 12070.

18 H. L. Tuller and S. R. Bishop, Annu. Rev. Mater. Res., 2011, 41, 369 . 
19 H. L. Tuller and A. S. Nowick, J. Phys. Chem. Solids, 1977, 38, 859.

20 M. P. Yeste, et al., Chem. Mater., 2006, 18, 2750.

21 S. R. Bishop, T. S. Stefanik and H. L. Tuller, Phys. Chem. Chem. Phys., 2011, 13, 10165.

22 The chemical expansion coefficient for CGO was extracted by fitting the entire non-stoichiometry range in Fig. 3.

23 S. R. Bishop, et al., J. Eur. Ceram. Soc., 2011, 31, 2351.

24 The $\mathrm{Zr}$ and Ce cations were randomly distributed in these configurations, consistent with the fluorite structure. No evidence of cation ordering was obtained from the HTXRD data, in agreement with this picture.

25 T. A. Lee, et al., J. Mater. Res., 2008, 23, 1105.

26 G. Zhou, et al., Catal. Today, 2007, 123, 86.

27 G. Balducci, et al., Catal. Lett., 1995, 33, 193.
28 G. Balducci, et al., J. Phys. Chem. B, 1998, 102, 557.

29 G. Liu, et al., J. Phys. Chem. B, 2001, 105, 7762.

30 J. A. Rodriguez, et al., J. Phys. Chem. B, 2003, 107, 3535.

31 G. Dutta, et al., Catal. Lett., 2006, 108, 165.

32 P. Li, I. W. Chen and J. E. Pennerhahn, Phys. Rev. B: Condens. Matter, 1993, 48, 10063.

33 P. Li, I. W. Chen and J. E. Pennerhahn, Phys. Rev. B: Condens. Matter, 1993, 48, 10074.

34 P. Li, I. W. Chen and J. E. Pennerhahn, Phys. Rev. B: Condens. Matter, 1993, 48, 10082.

35 V. Ruehrup and H.-D. Wiemhoefer, Z. Naturforsch., B: J. Chem. Sci., 2006, 61, 916.

36 X. Xie, et al., J. Mater. Chem., 2011, 21, 9570.

37 K. Kerman, B.-K. Lai and S. Ramanathan, J. Power Sources, 2012, 202, 120. 